\section{Dry Beriberi Manifesting as Acute Inflammatory Demyelinating Polyneuropathy in a Patient With Decompensated Alcohol-Induced Cirrhosis}

\author{
Amaninder Dhaliwal ${ }^{1}$, Jessica L. Larson ${ }^{2}$, Banreet S. Dhindsa ${ }^{3}$, Neil Bhogal ${ }^{3}$, Fedja A. Rochling ${ }^{3}$ \\ 1. Gastroenterology and Hepatology, University of South Florida Morsani College of Medicine, Tampa, USA 2. Internal \\ Medicine, University of Nebraska Medical Center, Omaha, USA 3. Gastroenterology and Hepatology, University of \\ Nebraska Medical Center, Omaha, USA
}

Corresponding author: Amaninder Dhaliwal,dramaninderdhaliwal@gmail.com
Review began 09/24/2020 Review ended 10/22/2020 Published 10/31/2020

\section{() Copyright 2020}

Dhaliwal et al. This is an open access article distributed under the terms of the Creative Commons Attribution License CC-BY 4.0., which permits unrestricted use, distribution, and reproduction in any medium, provided the original author and source are credited.
Categories: Gastroenterology, Oncology

Keywords: case report, beri beri, alcohol related cirrhosis, polyneuropathy

\section{Introduction}

Dry beriberi is a nutritional neuropathy that results from thiamine (vitamin B1) deficiency and is most commonly seen in individuals with a poor diet, alcoholism, or malabsorption syndromes. Thiamine is a cofactor that is essential for maintaining the myelin sheaths of neurons; however, the human body's storage only lasts up to 18 days. Dry beriberi commonly manifests as a symmetric sensorimotor neuropathy that primarily affects the distal extremities. In the majority of cases, it appears as a gradual, insidious onset but occasionally can appear rapidly resembling an acute inflammatory demyelinating polyneuropathy (AIDP). Dry beriberi is uncommon in developed countries, but its incidence is increasing alongside the growing prevalence of alcohol use. We present a case of dry beriberi manifesting as AIDP in a patient with decompensated alcohol-induced cirrhosis.

\section{Case Presentation}

A 28-year-old female from Nepal who recently immigrated to the United States presented to the ED with complaints of abdominal distention, fatigue, and six weeks of progressive bilateral lower extremity weakness. She was recently discharged from an outside hospital with a new diagnosis of decompensated liver cirrhosis with ascites. She reported drinking homemade alcoholic beverages daily while in Nepal but was unable to quantify the amount, given inconsistent history due to language and cultural barrier. She was taking thiamine $100 \mathrm{mg}$ oral daily, which was not continued on admission. On evaluation, she was extremely malnourished with a BMI of 14.6 due to lack of appetite and a diet consisting mostly of rice and beef broth soup. Physical exam demonstrated scleral icterus, abdominal distention with tenderness in the right upper quadrant, hepatosplenomegaly, and jaundice. Laboratory studies were notable for platelet count of 96,000/uL, albumin $2.8 \mathrm{~g} / \mathrm{dL}$, aspartate aminotransferase (AST) $52 \mathrm{U} / \mathrm{L}$, alanine aminotransferase (ALT) 30 $\mathrm{U} / \mathrm{L}$, total bilirubin $34.5 \mathrm{mg} / \mathrm{dL}$, international normalized ratio (INR) 1.8. Her initial model for end-stage liver disease (MELD) score was 26, and she was evaluated for liver transplantation. Other causes of cirrhosis, including viral, hemochromatosis, autoimmune, Wilson's disease, primary biliary cirrhosis, and primary sclerosing cholangitis, were excluded, and it was determined that she had alcohol-induced cirrhosis. Due to her extreme malnutrition and weakness, she was listed inactive on the transplant list.

The patient had bilateral lower extremity weakness and paresthesias preventing her from standing unassisted. She also demonstrated areflexia, tenderness to palpation, and pain with movement of lower extremities. Initial workup for weakness included vitamin B12, folate, and vitamin B6 levels. Vitamin B12 and folate returned within normal limits, but she was found to be deficient in vitamin B6 at $11.7 \mathrm{nmol} / \mathrm{L}$ and was started on replacement therapy. After 10 days of supplemental vitamin B6 and physical therapy, she had not improved, and neurology was consulted. An electromyogram was performed and consistent with severe sensorimotor axonal polyneuropathy with active denervation. Cerebrospinal fluid (CSF) analysis was also recommended and showed a slightly elevated protein at $47 \mathrm{mg} / \mathrm{dL}$ but not elevated enough to be consistent with Guillain-Barré syndrome (GBS). Additional labs were drawn, and she was found to have a low thiamine level at $55 \mathrm{nmol} / \mathrm{L}$ (normal: $70-80 \mathrm{nmol} / \mathrm{L}$ ). She was started on replacement therapy with slow 
improvement. By six months of aggressive thiamine replacement, she could walk 200 feet with a walker and stand without assistance.

\section{Discussion}

Thiamine is a water-soluble vitamin that is found in a variety of whole grains, nuts, and legumes. In the western world, many of our cereals and white grains are now fortified with thiamine making its deficiency uncommon. However, several populations are at risk of becoming thiamine deficient, including individuals with a poor diet, alcoholism, long-term parenteral nutrition use, or malabsorption syndromes.

Thiamine deficiency typically manifests as either Wernicke-Korsakoff syndrome or beriberi. Beriberi has historically been divided into two separate types: wet beriberi and dry beriberi. Wet beriberi affects the cardiovascular system, whereas dry beriberi impairs the central and peripheral nervous systems.

Both Wernicke-Korsakoff syndrome and dry beriberi affect the nervous system. This may be due to thiamine's use as a cofactor in the metabolism of carbohydrates to form adenosine triphosphate (ATP), generation of the neurotransmitter acetylcholine, and formation of myelin sheaths around axons [1]. Currently, there are three ways by which chronic alcohol intake can lead to thiamine deficiency. First, people with alcoholism can have a poor dietary intake of nutritious foods. Second, alcohol inhibits the absorption of thiamine across the gastrointestinal tract [2]. Lastly, alcohol leads to decreased utilization of thiamine in cells. Because only a small amount of thiamine is stored in the liver, a continuous supply of thiamine from the diet is needed. With limited tissue stores and inadequate nutrition, thiamine can be depleted in as little as 18 days [3].

Dry beriberi commonly develops as a gradual, insidious sensorimotor neuropathy in the distal extremities symmetrically. It can easily be confused with other types of neuropathy, including alcoholic neuropathy and AIDP, also known as GBS [4]. Our patient had a history of alcohol abuse and subsequently developed cirrhosis. She was extremely malnourished and underweight. She acutely developed profound weakness, areflexia, and paresthesia of lower extremities consistent with GBS. CSF analysis, however, was not consistent with GBS.

There have been several other case reports of dry beriberi resembling GBS [5-7] and even fewer case reports of patients with liver disease and concurrent dry beriberi [8,9]. Our case demonstrates the importance of having a high index of suspicion for thiamine deficiency in patients with alcohol-induced cirrhosis. By testing our patient's serum vitamin B1 level, the cause of her acute decline was discovered, and subsequently, she was started on thiamine replacement therapy. Had her thiamine level been tested sooner, she would have avoided unnecessary tests and shortened the length of her hospital stay. These patients show early response and can take up to six months for a full recovery, which is, unfortunately, not seen in all patients [10].

\section{Conclusions}

With the increasing rates of alcohol use, dry beriberi represents a potentially underdiagnosed cause of neuropathy. It is important to consider this diagnosis early in the evaluation of patients with history of excessive alcohol use who presents with symmetric sensorimotor neuropathy, as early replacement can prevent progression and potentially reverse neurological manifestations. Response can be variable and some patients can have irreversible damage.

\section{Additional Information \\ Disclosures}

Human subjects: Consent was obtained by all participants in this study. Conflicts of interest: In compliance with the ICMJE uniform disclosure form, all authors declare the following: Payment/services info: All authors have declared that no financial support was received from any organization for the submitted work. Financial relationships: All authors have declared that they have no financial relationships at present or within the previous three years with any organizations that might have an interest in the submitted work. Other relationships: All authors have declared that there are no other relationships or activities that could appear to have influenced the submitted work.

\section{References}

1. Osiezagha K, Ali S, Freeman C, et al.: Thiamine deficiency and delirium . Innov Clin Neurosci. 2013, 10:2632.

2. Martin PR, Singleton CK, Hiller-Sturmhöfel S: The role of thiamine deficiency in alcoholic brain disease Alcohol Res Health. 2003, 27:134-142.

3. MacLean LD, Rhode BM, Shizgal HM: Nutrition following gastric operations for morbid obesity. Ann Surg. 1983, 198:347-355. 10.1097/00000658-198309000-00011

4. Dimachkie MM, Barohn RJ: Guillain-Barré syndrome and variants. Neurol Clin. 2013, 31:491-510. 10.1016/j.ncl.2013.01.005 


\section{Cureus}

5. Howard AJ, Kulkarni O, Lekwuwa G, Emsley HC: Rapidly progressive polyneuropathy due to dry beriberi in a man: a case report. J Med Case Rep. 2010, 4:409. 10.1186/1752-1947-4-409

6. Riahi A, Mansour M, Bedoui I, Derbali H, Messelmani M, Zaouali J, Mrissa R: Acute beriberi neuropathy mimicking Guillain-Barré syndrome after a strict vegetarian diet. Iran J Neurol. 2017, 16:100-102.

7. Fu Liong H, Santhi DP, Shanthi V, Mohd Hanip R: Myanmarese neuropathy: clinical description of acute peripheral neuropathy detected among Myanmarese refugees in Malaysia. Int Sch Res Notices. 2014, 2014:187823. 10.1155/2014/187823

8. Naik M, Bhat T, Jalalie U, Bhat A, Gowhar W, Mir MF: Dry beriberi in a patient of acute fatty liver of pregnancy. Int J Nutr Pharmacol Neurol Dis. 2017, 7:18-20. 10.4103/2231-0738.199068

9. Di Marco S, Pilati L, Brighina F, Fierro B, Cosentino G: Wernicke-Korsakoff syndrome complicated by subacute beriberi neuropathy in an alcoholic patient. Clin Neurol Neurosurg. 2018, 164:1-4. 10.1016/j.clineuro.2017.11.006

10. Koike H, Ito S, Morozumi S, et al.: Rapidly developing weakness mimicking Guillain-Barre syndrome in beriberi neuropathy: two case reports. Nutrition. 2008, 24:776-780. 10.1016/j.nut.2008.02.022 